\title{
The characteristic time of glucose diffusion measured for muscle tissue at optical clearing
}

\author{
L M Oliveira, M I Carvalho, E M Nogueira and V V Tuchin
}

\begin{abstract}
The study of agent diffusion in biological tissues is very important to understand and characterize the optical clearing effects and mechanisms involved: tissue dehydration and refractive index matching. From measurements made to study the optical clearing, it is obvious that light scattering is reduced and that the optical properties of the tissue are controlled in the process. On the other hand, optical measurements do not allow direct determination of the diffusion properties of the agent in the tissue and some calculations are necessary to estimate those properties. This fact is imposed by the occurrence of two fluxes at optical clearing: water typically directed out of and agent directed into the tissue. When the water content in the immersion solution is approximately the same as the free water content of the tissue, a balance is established for water and the agent flux dominates. To prove this concept experimentally, we have measured the collimated transmittance of skeletal muscle samples under treatment with aqueous solutions containing different concentrations of glucose. After estimating the mean diffusion time values for each of the treatments we have represented those values as a function of glucose concentration in solution. Such a representation presents a maximum diffusion time for a water content in solution equal to the tissue free water content. Such a maximum represents the real diffusion time of glucose in the muscle and with this value we could calculate the corresponding diffusion coefficient.
\end{abstract}

\section{Introduction}

Laser and optical technologies in clinical practice represent a field with a significant volume of research in the past few decades. The study of light scattering in biological tissues is of major importance to improve and develop diagnostic and treatment biophotonic methodologies [1]. Imaging techniques represent an important method in clinical diagnosis and in 
some cases light scattering can be used to improve such techniques. A recent study using multispectral laser scattering images in pseudo colour has been presented as a possible method for rheumatism diagnosis [2]. On the other hand, light scattering can be a disadvantage in the improvement of some optical methods used in clinical practice [3]. As we see, light scattering in biological tissue is a very important phenomenon and computational models have been developed to perform simulations of light scattering in tissue [4].

One way to reduce light scattering and control the optical properties of tissues is to use the optical immersion clearing technique [5]. There are several diverse applications of optical clearing, depending on the type of biological tissue and the anatomical area. For instance, a study of the optical clearing of skull tissues has been presented recently, where the effectiveness of different optical clearing agents (OCAs) has been studied [6]. Other applications of optical clearing have been studied in ocular tissues [7] and blood [8]. Moreover, several methods have also been applied to study the efficiency of the optical clearing technique. Examples of these studies use backscattering circular polarized light [9] and optical coherence tomography (OCT) [10].

Several biological tissues are considered turbid since they present a collection of scattering elements with a high refractive index that are distributed through a background material with a lower refractive index. This difference in the refractive indices of the scattering elements and background material causes light scattering, and optical clearing is an effective way to reduce this difference. Optical clearing of tissues induces tissue dehydration and refractive index matching by agent inclusion in the interstitial space of the tissue [3]. By placing an OCA in the interstitial space of the tissue and reducing the water content, the refractive index difference between the scatterers and the interstitial liquid will be reduced and consequently, light scattering will decrease [5]. Skeletal muscle is an example of a biological tissue where a significant difference between the refractive indices of the scatterers and the interstitial liquid is observed [11]. In our previous research, we have created a model for rat muscle tissue, which contains completely dry scatterers with a refractive index of 1.584 distributed through the interstitial fluid, which is composed mainly of water with a refractive index of 1.333 [12]. Such a tissue can be subjected to optical clearing to reduce such a difference [13]. As an example, some studies and applications on muscle with imaging techniques could be improved if optical clearing is used $[14,15]$

The light scattering decrease is ultimately created by the refractive index matching that occurs at optical clearing if tissue dehydration is not strong [5]. The study of OCA diffusion behaviour into the tissue is important to understand

and describe the mechanisms involved at optical clearing and the time efficiency of treatment [16]. Several OCAs are known today and different biological tissues have been used to test them at optical clearing [16]. Aiming to study the way how agents penetrate the tissues, several researchers

have conducted studies to determine the diffusion properties of OCAs in the past few years. Several publications were presented with results for the diffusion time for some OCAs such as dimethyl sulfoxide [17], glucose [18-20], mannitol [18], sucrose [21], glycerol [22], lactose and fructose [21] in different biological tissues and phantoms. A study that was recently published provided results for glucose diffusion permeability in normal and cancerous oesophageal tissues [23]. Other studies to evaluate the efficiency of OCA concentration with tissue depth and the improved contrast of OCT images at higher tissue depths have also been reported [24-26]. Along with the experimental methods, some mathematical models have been developed to describe agent diffusion in biological tissues [3, 19, 27, 28].

With the objective of studying OCA diffusion into skeletal muscle, we have adopted a simple method to estimate the diffusion time and diffusion coefficient of glucose. Such methods are described thoroughly in the literature [3, 27, 29] By measuring the collimated transmittance spectra of tissue samples under treatment with different concentrated aqueous solutions of glucose we can model the time dependence of collimated transmittance for some particular wavelengths using equations similar to those that describe free diffusion. From the adjustments of experimental data with such a model we can determine the diffusion time for each treatment. The analysis of a representation of the diffusion time as a function of glucose concentration in the treatment solution allows the estimation of the true diffusion time of glucose in the refractive index matching mechanism of optical clearing.

Considering that glucose molecules have a free diffusion when entering into the interstitial space of muscle tissue, the literature indicates the equations that describe such a mechanism [3]. If we consider that a tissue sample has a slab form with thickness $d$, and that the agent diffuses through both surfaces of the slab at the same time, the diffusion is characterized by Fick's law [3,27]:

$$
\frac{\partial C_{\mathrm{a}}(x, t)}{\partial t}=D_{\mathrm{a}} \frac{\partial^{2} C_{\mathrm{a}}(x, t)}{\partial x^{2}} .
$$

Sucn an equation alıows the determination or tne time dependence of OCA concentration $C_{\mathrm{a}}$ at any unidirectional position $x$ between the two surfaces. In equation (1), $D_{\text {a }}$ represents the diffusion coefficient of the OCA in the tissue. When the OCA diffusion takes place through the two surfaces of the tissue slab, the diffusion time of OCA is related to the diffusion coefficient in the following way [3, 27]:

$$
\tau=\frac{d^{2}}{\pi^{2} D_{\mathrm{a}}} .
$$

If the volume of solution used is significantly higher than the volume of the slab (e.g., 10x), we can determine the amount of dissolved matter $m_{t}$ at an instant $t$ to its equilibrium value $m_{\infty}$ according to the following definition [3]:

$$
\begin{aligned}
\frac{m_{t}}{m_{\infty}}= & \frac{\int_{0}^{d} C_{\mathrm{a}}(x, t) \mathrm{d} x}{C_{G 0} d} \\
= & 1-\frac{8}{\pi^{2}}\left[\exp \left(-\frac{t}{\tau}\right)+\frac{1}{9} \exp \left(-\frac{9 t}{\tau}\right)\right. \\
& \left.+\frac{1}{25} \exp \left(-\frac{25 t}{\tau}\right)+\cdots\right] .
\end{aligned}
$$




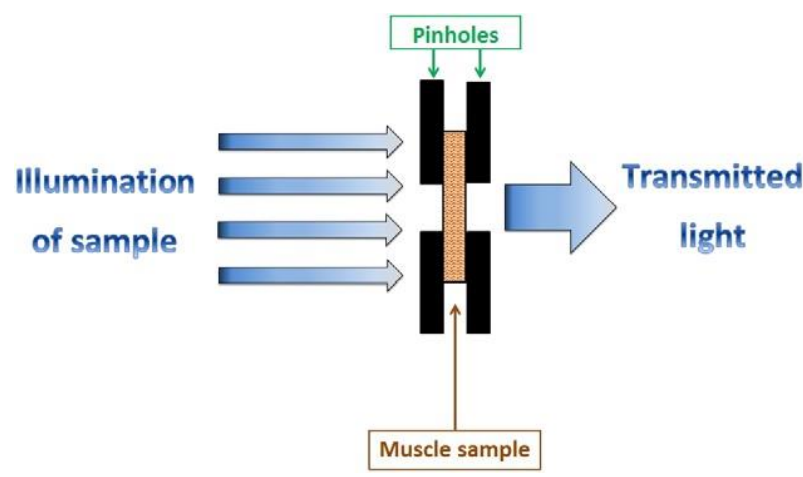

Figure 1. Measurement assembly to obtain collimated transmittance.

The ratio in equation (3) defines the volume averaged concentration of an agent $C_{\mathrm{a}}(t)$ within the slab at time $t$. As a first-order approximation, this equation has a solution given by $[3,5]$ :

$$
C_{\mathrm{a}}(t)=\frac{1}{d} \int_{0}^{d} C_{\mathrm{a}}(x, t) \mathrm{d} x \cong C_{a 0}\left[1-\exp \left(-\frac{t}{\tau}\right)\right] .
$$

From equation (4), we see a relation between the time dependence of OCA concentration within the sample and its characteristic diffusion time $T$. When using that diffusion time $T$ in equation (2) we can calculate the corresponding diffusion coefficient. These are the two parameters that we want to estimate for glucose in skeletal muscle.

\section{Materials and methods}

To perform our study, we have selected the skeletal muscle from the abdominal wall of rat-species Wistar Han. We have sacrificed one animal and retrieved the entire muscle block from the abdominal wall. From that block and using a cryostat, we have sliced eight samples with approximately circular form of $10 \mathrm{~mm}$ in diameter and $0.5 \mathrm{~mm}$ in thickness.

Using a measurement assembly like the one presented in cross-section in figure 1 , we have started by measuring the collimated transmittance of the natural sample.

The pinholes presented in figure 1 have a circular form to fix the sample. They have a central hole with $1 \mathrm{~mm}$ diameter to allow the passage of light. After measuring the natural sample, we have immersed the sample in an aqueous solution containing $20 \%$ of glucose. The agent is delivered by a lateral opening that is not seen in the cross-section representation in figure 1. The collimated transmittance measurements were done every second for the whole spectrum from 400 to $1000 \mathrm{~nm}$ (for each second 37 spectra were averaged) for a period of $30 \mathrm{~min}$. Such a procedure was repeated for the treatments with other samples and solutions containing glucose in higher concentrations. The concentrations of glucose in the solutions used were $20 \%, 25 \%, 30 \%, 35 \%$, $40 \%, 45 \%, 50 \%$ and $54 \%$. The total number of samples under study was eight, one for each treatment.

After completing the measurements the spectral data were processed. For each treatment, we have retrieved and represented the time variation of collimated transmittance for different wavelengths. The selected wavelengths were 400 , 500, 600, 700, 800, 900 and $1000 \mathrm{~nm}$. After representation of the time dependences observed in each treatment, for better precision in calculations, we have selected only wavelengths at each $20 \mathrm{~nm}$ between 600 and $800 \mathrm{~nm}$, where the tissue spectral profile is mostly defined by scattering. For each of the time variations observed for those wavelengths we have displaced the natural transmittance to zero and normalized each entire data set to its highest value. In this way the time dependence observed for each particular wavelength is contained within the interval $0-1$. All the displaced and normalized data sets were adjusted with lines with equations of the form:

$$
T_{\mathrm{c}}(\lambda)=1-\exp \left(-\frac{t}{\tau}\right) .
$$

When making these adjustments we obtained the $T$ values for each adjustment. If we see that the second member of equation (5) is the same as the term in parenthesis for the second member of equation (4), the $T$ value in equation (5) represents the diffusion time of the combined fluxes in each treatment (water out of and glucose into the tissue). To treatment (water out of and glucose into the tissue). To determine this diffusion time of the mixed fluxes in one treatment we have used the diffusion time values obtained for each wavelength to calculate the mean and standard deviation value for that treatment. With the mean diffusion time values obtained for each treatment we have represented the mean diffusion time as a function of the glucose concentration in solution used in the various treatments studied. The analysis of that representation allows one to retrieve valuable information and to estimate the true diffusion time of glucose in skeletal muscle.

\section{Results}

Using the experimental assembly presented in figure 1, we have measured the collimated transmittance in the various treatments with solutions containing the above indicated concentrations of glucose. The spectra were collected during the various treatments for a $30 \mathrm{~min}$ period.

Considering wavelengths between 400 and $1000 \mathrm{~nm}$, we represent in figure 2 the time variations of collimated transmittance for some of the treatments.

Similar time dependences were also obtained for the other treatments. As we can see from figure 2, for the treatment with the lowest concentration of glucose in solution (20\%) we have a fast diffusion and a significantly decreasing saturation regime. This means that the immersion solution contains a great amount of water compared with the tissue water content. In that case, possibly two water fluxes are created, one directed into the tissue, caused by differences between the water concentration inside and outside, and another one directed out of the tissue, caused by the osmotic action of glucose. Therefore, because of the strong involvement of small water molecules in the diffusion process, the total time response of the diffusion process is short (fast diffusion). After the saturation regime is reached, the tissue needs to 


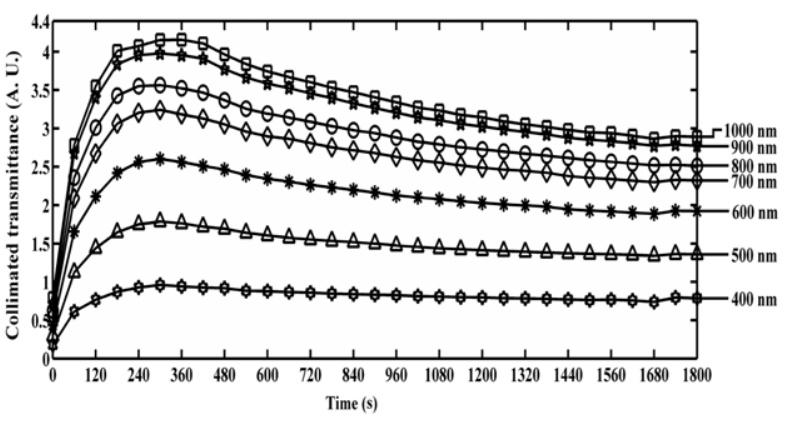

(a)

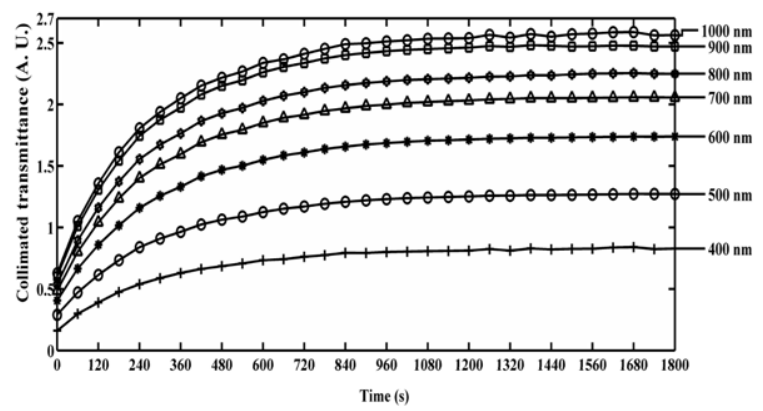

(c)

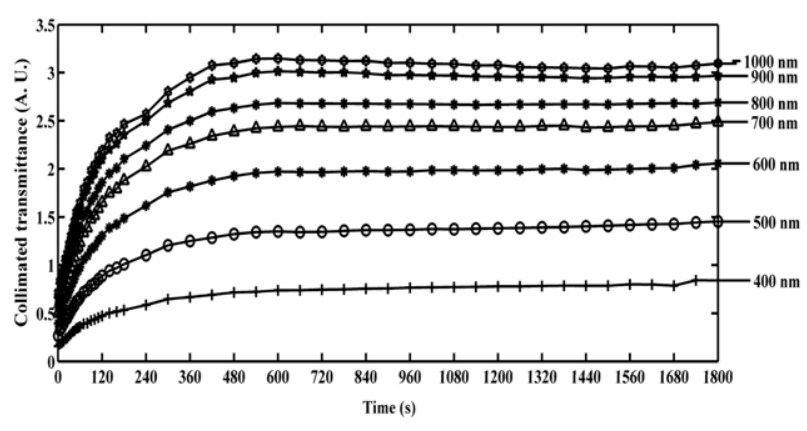

(b)

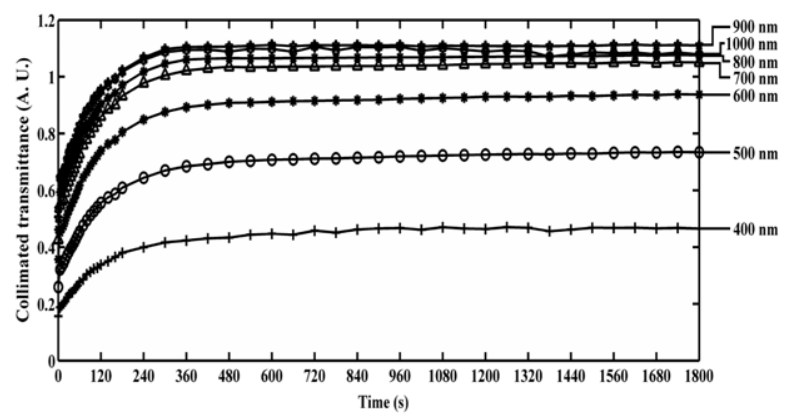

(d)

Figure 2. Time dependence of collimated transmittance observed for treatments with: (a) glucose 20\%; (b) glucose $35 \%$; (c) glucose $40 \%$; (d) glucose $50 \%$.

compensate the partial dehydration caused by the impact of glucose, and so it starts to receive water from the solution to create a balance and tissue swelling may be created with some decay of the collimated transmittance for longer times. Such a decreasing behaviour during saturation is not seen in the other treatments presented in figure 2. Considering the various solutions used, as the glucose concentration increases (figures 2(b) and (c)), the water content in the solution becomes similar to the free water content in the tissue and the water flux decreases, becoming close to zero at a glucose concentration around $40 \%$ (figure 2(c)). That means that only glucose diffuses freely in the system, and the diffusivity parameters depend only on the diffusion parameters of the larger glucose molecules, thus we see a maximal value of the measured diffusion time (figure 2(c)). However, at still higher glucose concentration we see once again a decreased diffusion time $T$ (figure 2(d)), because water is once again involved in the diffusion process, with the main flux directed from the tissue to the surrounding solution due to glucose hyperosmolarity.

Selecting the data sets observed for wavelengths between 600 and $800 \mathrm{~nm}$ we have made the above described normalization for each wavelength and treatment, then using equation (5) we have calculated corresponding values of the diffusion time. For a particular glucose concentration, the mean and standard deviation of the diffusion time, measured at 11 wavelengths, was calculated. Table 1 lists the various concentrations of glucose in solution used and the calculated mean diffusion time and standard deviation for

each treatment:

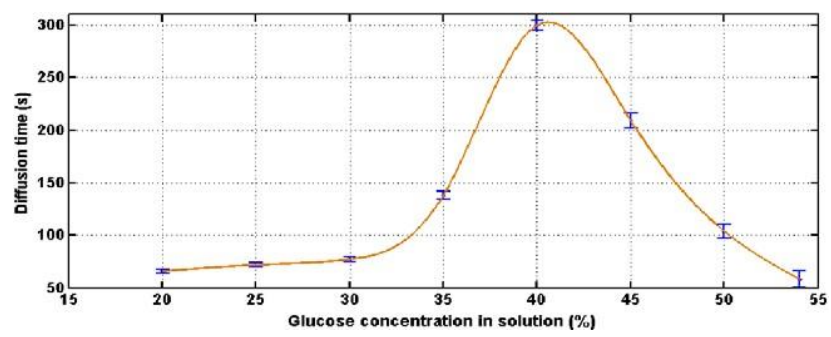

Figure 3. Diffusion time of glucose as a function of glucose concentration in solution.

Using the values in table 1 we represent in figure 3 the mean diffusion time of glucose as a function of glucose concentration in the solutions used in the various treatments. Figure 3 also contains the fit to the data made with a natural spline.

We can see in figure 3 that the fit made to the data presents a maximum for a concentration of $40.5 \%$. This maximum indicates that an aqueous solution with that concentration of glucose would have the same amount of water as the free water in the tissue. In such a case no water flux occurs, just glucose flux into the tissue. From the plot in figure 3, we have obtained a diffusion time of $302.9 \mathrm{~s}$ for glucose. This value represents the true diffusion time of glucose in the skeletal muscle. Using this value in equation (2), we can calculate the diffusion coefficient of glucose in muscle:

$$
D_{\mathrm{a}}=\frac{d^{2}}{\pi^{2} \tau}=\frac{0.05^{2}}{\pi^{2} \times 302.9}=8.36 \times 10^{-7} \mathrm{~cm}^{2} \mathrm{~s}^{-1} \text {. }
$$


Table 1. Mean and standard deviation values for the diffusion time of glucose for the different treatments studied $(n=11)$.

\begin{tabular}{|c|c|c|c|c|c|c|c|c|}
\hline $\begin{array}{l}\text { Concentration of glucose in } \\
\text { solution }(\%)\end{array}$ & 20 & 25 & 30 & 35 & 40 & 45 & 50 & 54 \\
\hline Mean diffusion time (s) & 65.9 & 72.0 & 77.3 & 138.3 & 300.0 & 209.4 & 103.6 & 58.4 \\
\hline SD (s) & 1.8 & 2.0 & 2.2 & 4.3 & 4.9 & 7.4 & 7.0 & 7.9 \\
\hline
\end{tabular}

In this calculation we have used a slab thickness of $0.5 \mathrm{~mm}$.

In this way we have estimated the diffusion properties of glucose in skeletal muscle from the spectral measurements of the collimated transmittance.

\section{Conclusions}

As we can see from the results presented above, we were able to use a simple method to identify the diffusion time and diffusion coefficient of glucose in skeletal muscle during optical clearing. From our measurements and analysis we could also estimate the tissue as having a free water content of $59.5 \%$. This value is smaller than the $75.6 \%$ of total water content for the skeletal muscle of rat [30]. This means that the remaining $16.1 \%$ of water is bound to the tissue components and does not participate in the dehydration mechanism at optical clearing, at least on a timescale of $30 \mathrm{~min}$. After estimating these properties, we can now use the diffusion coefficient in equation (4) to determine the time dependence of glucose concentration inside the muscle for any treatment with a solution of known glucose concentration.

After performing this study, it becomes imperative to perform similar studies with different OCAs to determine their corresponding diffusion properties in the skeletal muscle. As single wavelength measurements of the collimated transmittance are sufficient for quantification of the diffusion parameters, brighter light sources, such as lasers and LEDs, could be used to make measurements for thicker tissue samples.

\section{Acknowledgments}

The authors would like to thank the following institutions for their help in preparation of the samples and resources made available to perform the measurements: CIETI - Centro de Inovação em Engenharia e Tecnologia Industrial, ISEPInstituto Superior de Engenharia do Porto, Portugal and LAIMM - Laboratório de Apoio à Investigação em Medicina Molecular, Departamento de Biologia Experimental, Faculdade de Medicina da Universidade do Porto, Portugal.

VVT is grateful for support from the RF Government contracts 11.519.11.2035, 14.B37.21.0563, 14.B37.21.0728, and 14.512.11.0022; project No. 1.4.09; FiDiPro, TEKES Program (40111/11), Finland; RF President's grant 1177.2012.2 'Scientific Schools'.

\section{References}

[1] Tuchin V V 2007 Tissue Optics: Light Scattering Methods and Instruments for Medical Diagnosis 2nd edn (Bellingham, WA: SPIE)

[2] Zabarylo U and Minet O 2009 Pseudo colour visualization of fused multispectral laser scattering images for optical diagnosis of rheumatoid arthritis Laser Phys. Lett. 773-7

[3] Tuchin V V 2006 Optical Clearing of Tissues and Blood (Bellingham, WA: SPIE)

[4] Tanev S, Tuchin V V and Paddon P 2006 Light scattering effects of gold nanoparticles in cells: FDTD modeling Laser Phys. Lett. 3 594-8

[5] Tuchin V V 2005 Optical immersion as a new tool for controlling the optical properties of tissues and blood Laser Phys. 15 1109-36 (www.maik.ru/full/lasphys/05/8/ lasphys8_05p1109full.pdf)

[6] Wang J, Zhang Y, Xu T H, Luo Q M and Zhu D 2012 An innovative transparent cranial window based on skull optical clearing Laser Phys. Lett. 9469-73

[7] Alekhin A A et al 2010 In vitro femtosecond laser subsurface micro-disruption inside human cornea and pre-cleared sclera Laser Phys. Lett. 7 463-6

[8] Zhernovaya O S, Tuchin V V and Meglinski I V 2008 Monitoring of blood proteins glycation by refractiveindex and spectral measurements Laser Phys. Lett. 5460-4

[9] Macdonald C and Meglinski I 2010 Backscattering of circular polarized light from a disperse random medium influenced by optical clearing Laser Phys. Lett. 8324-8

[10] Proskurin S G and Meglinski I V 2007 Optical coherence tomography imaging depth enhancement by superficial skin optical clearing Laser Phys. Lett. 4 824-6

[11] Oliveira L M, Lage A S, Pais Clemente M A and Tuchin V V 2010 Rat muscle opacity decrease due to the osmosis of a simple mixture J. Biomed. Opt. 15055004

[12] Oliveira L M, Lage A S, Pais Clemente M A and Tuchin V V 2009 Optical characterization and composition of abdominal wall muscle from rat Opt. Lasers Eng. 47 667-72

[13] Oliveira L, Carvalho M I, Nogueira E and Tuchin V V 2013 Optical measurements of rat muscle samples under treatment with ethylene glycol and glucose J. Innovative Opt. Health Sci. 61350012

[14] Minet O, Zabarylo U and Beuthan J 2005 Deconvolution of laser based images for monitoring rheumatoid arthritis Laser Phys. Lett. 2 556-63

[15] Minet O, Gajewski H, Griepentrog J A and Beuthan J 2007 The analysis of laser light scattering during rheumatoid arthritis by image segmentation Laser Phys. Lett. 4604-10

[16] Genina E A, Bashkatov A N and Tuchin V V 2010 Tissue optical immersion clearing Expert Rev. Med. Devices 7 825-42

[17] Liu P, Huang Y, Guo Z, Wang J, Zhuang Z and Liu S 2013 Discrimination of dimethyl sulphoxide diffusion coefficient in the process of optical clearing by confocal micro-Raman spectroscopy J. Biomed. Opt. 18020507 
[18] Bashkatov A N, Genina E A, Sinichkin Y P, Kochubey V I, Lakodina N A and Tuchin V V 2003 Glucose and mannitol diffusion in human dura matter Biophys. J. 85 3310-8

[19] Bertram R and Pernarowski M 1998 Glucose diffusion in pancreatic islets of Langerhans Biophys. J. 74 1722-31

[20] Ghosn M G, Carbajal E F, Befrui N A, Tuchin V V and Larin K V 2008 Differential permeability rate and percent clearing of glucose in different regions in rabbit sclera J. Biomed. Opt. 13021110

[21] Ribeiro A C F, Ortona O, Simões S M N, Santos C I A V, Prazeres P M R A, Valente A J M, Lobo V M M and Burrows H D 2006 Binary mutual diffusion coefficients of aqueous solutions of sucrose, lactose, glucose, and fructose in the temperature range from (298.15 to 328.15$) \mathrm{K}$ J. Chem. Eng. Data 51 1836-40

[22] Zhong H Q, Guo Z Y, Wei H J, Zeng C C, Xiong H L, He Y H and Liu S H 2010 Quantification of glycerol diffusion in human normal and cancer breast tissues in vitro with optical coherence tomography Laser Phys. Lett. 7315-20

[23] Zhao Q L et al 2011 Quantifying glucose permeability and enhanced light penetration in ex vivo human normal and cancerous esophagus tissues with optical coherence tomography Laser Phys. Lett. 871-7
[24] Ghosn M G, Carbajal E F, Befrui N A, Tuchin V V and Larin K V 2008 Concentration effect on the diffusion of glucose in ocular tissues Opt. Lasers Eng. 46911-4

[25] Vargas G, Chan E K, Barton J K, Rylander H G and Welch A J 1999 Use of an agent to reduce scattering in skin Lasers Surg. Med. $24133-41$

[26] Larina I V, Carbajal E F, Tuchin V V, Dickinson M E and Larin K V 2008 Enhanced OCT imaging of embryonic tissue with optical clearing Laser Phys. Lett. 5476-9

[27] Bashkatov A N, Genina E A and Tuchin V V 2009 Measurement of glucose diffusion coefficients in human tissues Handbook of Optical Sensing of Glucose in Biological Tissues and Fluids ed V V Tuchin (Boca Raton, FL: Taylor and Francis)

[28] Stolnitz M M, Bashkatov A N, Genina E A and Tuchin V V 2007 Mathematical modeling of clearing liquid penetration into the skin Proc. SPIE $\mathbf{6 5 3 5} 653520$

[29] Bashkatov A N, Genina E A and Tuchin V V 2002 Optical immersion as a tool for tissue scattering properties control Perspectives in Engineering Optics ed K Singh and V K Rastogi (New Delhi: Anita) pp 313-34

[30] Reinoso R F, Telfer B A and Rowland M 1997 Tissue water content in rats measured by desiccation J. Pharmacol. Toxicol. Methods 38 87-92 